Classification

Physics Abstracts

$75.40-75.60 \mathrm{E}-75.50 \mathrm{~K}$

\title{
Critical behaviour in an insulating spin glass : field expansion and scaling
}

\author{
P. Beauvillain, C. Chappert and J. P. Renard \\ Institut d'Electronique Fondamentale (*), Université Paris XI, Bât. 220, \\ 91405 Orsay Cedex, France
}

(Reçu le 20 février 1984, accepté le 14 mai 1984)

\begin{abstract}
Résumé. - Nous avons mesuré la susceptibilité magnétique alternative en champ magnétique appliqué $H$ et l'aimantation statique d'un verre de spin isolant : l'aluminosilicate de manganèse amorphe au voisinage de sa température de gel $T_{\mathrm{f}}\left(T_{\mathrm{f}}=2,95 \mathrm{~K}\right)$. Nous pouvons décrire les mesures de susceptibilité alternative en champ à l'aide d'un développement en puissances paires de $H$ et nous avons déterminé la variation thermique des quatre premiers termes de ce développement. La partie non linéaire de la susceptibilité alternative peut être décrite par une fonction universelle avec les exposants $\gamma=3,1 \pm 0,1$ et $\beta=1,4 \pm 0,1$. Nous avons également mesuré l'aimantation statique en champ magnétique très faible $(0,01$ Oe-10 Oe) à l'aide d'un magnétomètre à SQUID et nous avons observé des phénomènes de relaxation dans l'aimantation refroidie sous champ. Au voisinage immédiat de la température de gel $\left(t \sim 10^{-2}\right)$. La partie non linéaire de l'aimantation statique peut s'interpréter à l'aide de la fonction universelle précédente.
\end{abstract}

\begin{abstract}
The amorphous manganese aluminosilicate with 15 at $\% \mathrm{Mn}$ is a spin glass with a freezing temperature $T_{\mathrm{f}}=2.95 \mathrm{~K}$. We have precisely measured slightly above $T_{\mathrm{f}}$ its ac susceptibility in a constant applied field $H$ and its low field dc magnetization. The field cooled magnetization displays interesting relaxation phenomena close above and below $T_{\mathrm{f}}$. The ac susceptibility data can be fitted with a series expansion in even powers of $H$ and we determined the thermal variation of the first four terms of the series. The non linear part of the ac susceptibility can be described above $T_{\mathrm{f}}$ by a universal scaling function with two exponents : $\gamma=3.1 \pm 0.1$ and $\beta=1.4 \pm 0.1$. By means of numerical integration on this scaling function we calculated the non linear part of the equilibrium dc magnetization slightly above $T_{\mathrm{f}}$ : the agreement with our precise SQUID measurements is excellent for $\left(T-T_{\mathrm{f}}\right) / T_{\mathrm{f}} \gtrsim 10^{-2}$.
\end{abstract}

\section{Introduction.}

The mean field (MF) theory of the spin glasses (SG) predicts a transition from the paramagnetic (P) phase to the SG phase characterized by a divergence of the non linear magnetization [1]. More precisely, in the field series expansion of the equilibrium magnetization in the $\mathrm{P}$ phase,

$$
M(H, T)=\chi_{0}(T) H-a_{1}(T) H^{3}+(-1)^{n} a_{n}(T) H^{2 n+1}+\cdots
$$

(*) Laboratoire associé au C.N.R.S. 
the coefficients $a_{n}(T)$ diverge at a well defined temperature $T_{\mathrm{f}}$, while $\chi_{0}$ remains finite at $T_{\mathrm{f}}$. Slightly above $T_{\mathrm{f}}, a_{1} \simeq\left(T-T_{\mathrm{f}}\right)^{-1}, a_{2} \simeq\left(T-T_{\mathrm{f}}\right)^{-3}$.

A careful study of $M(H, T)$ at $T \rightarrow T_{\mathrm{f}}$ and $H \rightarrow 0$ would provide an experimental test of the MF theory. This was done in AgMn : $10.6 \%$ by Monod and Bouchiat [2] who observed a qualititative agreement with the MF predictions. Unfortunately, the lack of precision in $M / H$ at low values of $H$ ruled out a quantitative determination of $a_{1}$ near $T_{\mathrm{f}}$. In a similar study on amorphous GdAl, Barbara et al. [3] used the following phenomenological expression of $M(H, T)$

$$
M(H, T)=\chi_{0}(T) \cdot H-\chi_{1}(T) \cdot H^{a(T)}
$$

which they assumed to be valid in a relatively large field range. In addition, these authors attempted to represent their experimental data for $\chi_{n l}(H, T)=\chi_{0}-M / H$ by a scaling function. In their recent work on $\mathrm{CuMn}: 1$ at \%, Omari et al. [4] succeeded in determining the temperature dependence of the coefficients $a_{1}$ and $a_{2}$ defined by equation (1), in the temperature range 1:1 $T_{1}$ to $4 T_{\mathrm{f}}$. They also fitted all their experimental data in the same temperature range and for $H$ up to $60 \mathrm{kOe}$ by a scaling function. In a previous work [5], we reported on the ac susceptibility and the equilibrium magnetization of an insulating spin glass : amorphous manganese aluminosilicate. In this SG with short range interactions, the behaviour of $M(H, T)$ is rather similar to the one observed in the metallic SG with RKKY interactions.

So far, no precise measurements have been done in SG which are both at low field values and very close to the freezing temperature. The major difficulty at low $H$ is to determine accurately the ratio $M / H$ and its limit $\chi_{0}$ for $H \rightarrow 0$. An improvement by at least one order of magnitude is achieved here by measuring directly the effects of the field on the ac susceptibility. In the absence of dynamical effects, $\chi_{\mathrm{ac}}$ is given by :

$$
\chi_{\mathrm{ac}}(H, T)=\frac{\partial M}{\partial H}=\chi_{0}-3 a_{1} H^{2}+(-1)^{n}(2 n+1) a_{n} H^{2 n}+\cdots .
$$

When approaching $T_{\mathrm{f}}, \chi_{\mathrm{ac}}(H=0)$ starts to differ from $\frac{\partial M}{\partial H}(H=0)$ because of the slowing down of the magnetization relaxation; on the other hand, the non linear part of $M(H)$ grows and can be detected by low field SQUID magnetization measurements.

These ideas have been used for performing a detailed study of the singularity of the non linear magnetization in amorphous manganese aluminosilicate. The experiments have been done on a sample of amorphous $(\mathrm{MnO})_{x}\left(\mathrm{Al}_{2} \mathrm{O}_{3}\right)_{y}\left(\mathrm{SiO}_{2}\right)_{z}$ with an $\mathrm{Mn}$ atomic concentration of about $15 \%$, prepared by Dr. K. Knorr from the Institut für Kristallographie of Tübingen, FRG. The freezing temperature of this sample observed on the field cooled magnetization at low field is $T_{\mathrm{f}}=2.95 \mathrm{~K}$. The ac susceptibility and dc SQUID experimental set-up have been previously described [5].

\section{2. ac susceptibility.}

The ac susceptibility has been measured at $69 \mathrm{~Hz}$ in the temperature range $3-4.2 \mathrm{~K}$. The amplitude of the measuring field was 3 Oe. The superimposed static field was varied in the range 0-500 Oe. The sensitivity amounted to $2 \times 10^{-7}$.emu cgs.

2.1 INITIAL SUSCEPTIBILITY. - The initial $(H=0)$ ac susceptibility versus $T$ is shown in figure 1 and compared to the static field cooled magnetization in 0.1 Oe. No difference is observed between 3.25 and $4.2 \mathrm{~K}$. In this temperature range, the initial susceptibility is well fitted by $\chi=A /(T-\theta)$ with $\theta=-1.23(4) \mathrm{K}$ and $A=4.097 \times 10^{-3} \mathrm{emu} \mathrm{cgs} / \mathrm{g}$. In table I we give a precise comparison between the ac and static determination of the initial susceptibility. 


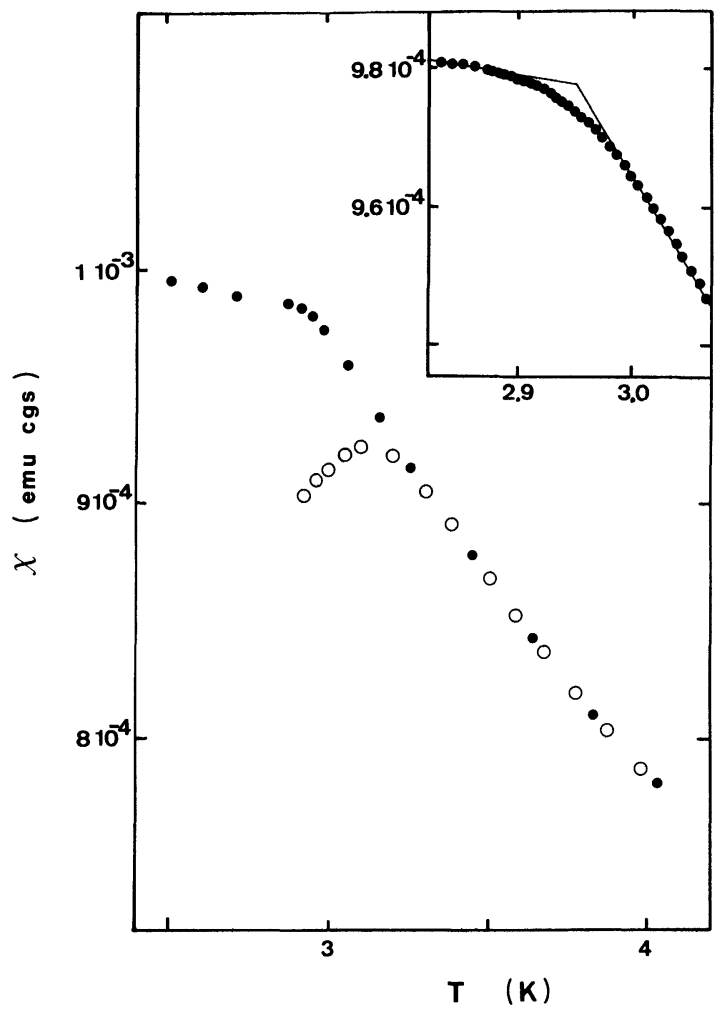

Fig. 1. - ac susceptibility (open circles) at $69 \mathrm{~Hz}$ and $M / H$ (black circles) versus temperature. $M$ is the field cooled magnetization measured in $H=0.1 \mathrm{Oe}$.

Table I. - Precise comparison between initial ac susceptibility and static field cooled magnetization in $0.1 \mathrm{Oe}$ in the temperature range $3.1-4.2 \mathrm{~K}$.

\begin{tabular}{|c|c|c|}
\hline \multicolumn{2}{|c|}{$T(\mathbf{K})$} & $\begin{array}{c}\chi \times(T+1.234) \\
\left(10^{-3} \mathrm{emu} \operatorname{cgs} / \mathrm{g} \times \mathrm{K}\right)\end{array}$ \\
\hline \multirow{2}{*}{ ac } & 4.211 & 4,102 \\
& 4.105 & 4,100 \\
& 3.999 & 4,099 \\
& 3.901 & 4,097 \\
& 3.802 & 4,092 \\
& 3.701 & 4,093 \\
& 3.599 & 4,095 \\
& 3.400 & 4,097 \\
& 3.300 & 4,095 \\
& 3.200 & 4,071 \\
& 3.102 & 3,998 \\
\hline \multirow{2}{*}{$\mathrm{dc}$} & 4.235 & 4,098 \\
& 3.597 & 4,092 \\
& 3.497 & 4,097 \\
& 3.149 & 4,097 \\
\hline
\end{tabular}


It should be pointed out that the magnetization relaxation time decreases in an applied field. Thus $\chi_{\mathrm{ac}}$ is equal to $\partial M / \partial H$ for $T>3.25 \mathrm{~K}$.

2.2 ac SUSCEPTIBILITY IN AN APPLIED FIELD. - The isothermal field variation of the ac susceptibility has been measured for eleven equally spaced values of temperature between 3.2 and $4.2 \mathrm{~K}$. Some of these experimental curves shown in figure 2 clearly illustrate the occurrence of a singularity in the non linear susceptibility of a spin glass near its freezing temperature.

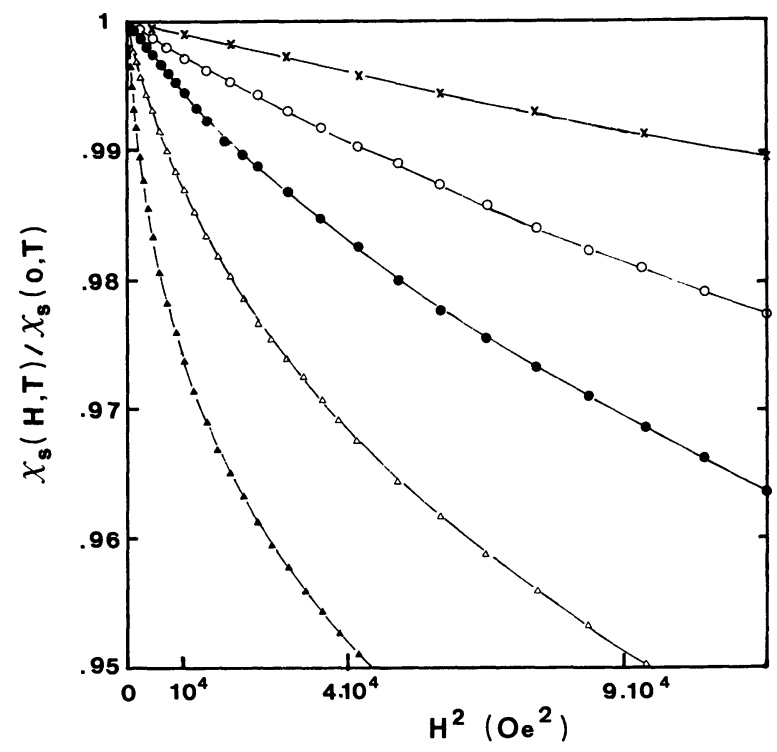

Fig. 2. - Non linear ac susceptibility $\chi_{\mathrm{s}}(H, t)$ versus $H^{2}$ with $\chi_{\mathrm{s}}=\chi_{0}(t)-\frac{\partial M}{\partial H}$. Crosses $: t=0.43$; open circles $: t=0.29$; black circles $: t=0.22$; open triangles $: t=0.15$; black triangles $: t=0.08$.

The data were compared to the series expansion (3) and also to the empirical law (2) by means of least square fits. The following procedure has been used : at a given temperature, the fit is performed for the data restricted to the field range 0 to $H_{\text {lim }}$. The variance of the fit is then studied as a function of $H_{\text {lim. }}$. It is observed that the variance is fairly constant for small values of $H_{\text {lim }}$ and then increases rapidly when $H_{\text {lim }}$ exceeds a value $H_{\max }(T)$. We first compared the fits to the empirical law (2) and to the series expansion (3) restricted to the first three terms. The variance is close to 1 in both fits but the field extension $H_{\max }$ is greater with equation (3) for all temperatures. We thus considered that (3) provides a better description of the experimental data. A further improvement was obtained by extending the fit to the fourth term of (3). The three coefficients $a_{1}(T), a_{2}(T), a_{3}(T)$ are well described by the power law

$$
a_{n}(T)=b_{n} t^{-x_{n}}
$$

where $t$ is the reduced temperature $t=\left(T-T_{\mathrm{f}}\right) / T_{\mathrm{f}}\left(T_{\mathrm{f}}=2.95 \mathrm{~K}\right)$ and $0.08<t<0.43$ (Fig. 3). The experimental values of the amplitude and exponents are :

$$
\begin{aligned}
& b_{1}=1.54 \times 10^{-12} ; b_{2}=1.12 \times 10^{-20} \quad \text { and } \quad b_{3}=6.65 \times 10^{-29} \mathrm{emu} \mathrm{cgs} / \mathrm{g} \\
& x_{1}=3.1 \pm 0.1 ; \quad x_{2}=7.6 \pm 0.4 ; \quad x_{3}=12 \pm 1 .
\end{aligned}
$$




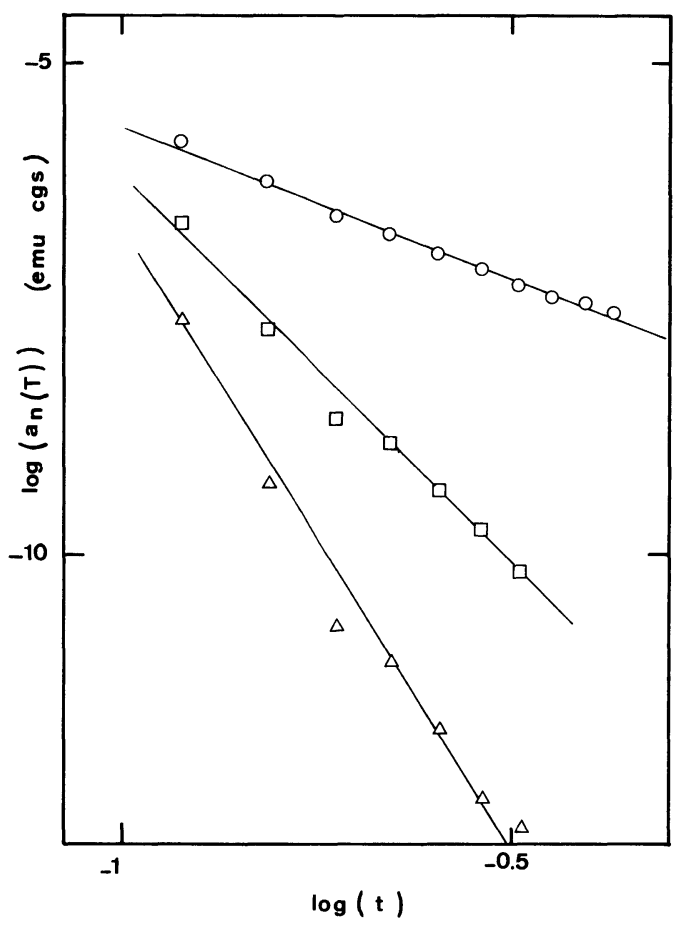

Fig. 3. - Thermal variation of $a_{n}(T)$. The $a_{n}(T)$ are the coefficients of series expansion (1); $n=1$ : circles ; $n=2:$ squares $;=3:$ triangles.

The value of the exponent $\gamma$ (or $\left.x_{1}\right)=3.1$ characterizing the temperature divergence of the first term of the non linear magnetization is consistent with those obtained on metallic spin glasses with long range RKKY interactions [3, 4]. We can also see in figure 3 that near $T_{\mathrm{f}}\left(t \sim 10^{-1}, H=34 \mathrm{Oe}\right)$ the three terms $a_{n}(T)$ are of the same order of magnitude. This means that in the series expansion (3), more and more terms must be taken into account when approaching $T_{\mathrm{f}}$.

2.3 EXPERIMENTAL TEST OF THE SCALING FUNCTION FOR $t>1 \times 10^{-1}$. - In order to take into account all our experimental data in the whole field range, we have tried to describe our susceptibility measurements with the scaling function $[3,5]$

$$
\frac{\chi_{\mathrm{s}}}{t^{\beta}}=f\left[\frac{H^{2}}{t^{\gamma+\beta}}\right]=g\left[\frac{H}{t^{\frac{\gamma+\beta}{2}}}\right]
$$

where $\chi_{\mathrm{s}}$ is the non linear susceptibility $\chi_{\mathrm{s}}=\chi_{0}-\frac{\partial M}{\partial H}$ and $\gamma, \beta$ two adjustable parameters. We have obtained a remarkable agreement between equation (5) and our data (Fig. 4) with $\gamma=3.1 \pm 0.1$ and $\beta=1.4 \pm 0.1$. Expanding (5) in powers of $H^{2} / t^{\gamma+\beta}$ leads to :

$$
\frac{\partial M}{\partial H}=\chi_{0}(T)-3 b_{1} t^{-\gamma} H^{2}+5 b_{2} t^{-(2 \gamma+\beta)} H^{4}-7 b_{3} t^{-(3 \gamma+2 \beta)} H \ldots
$$




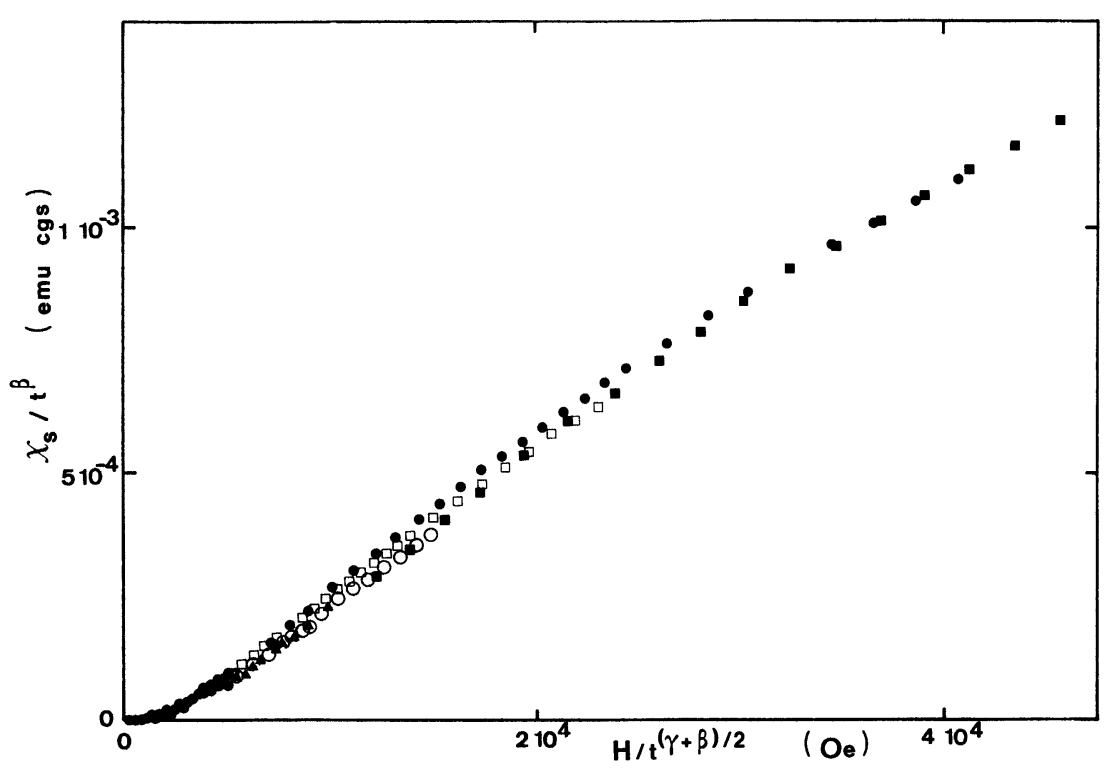

Fig. 4. - Scaling function $g(x)=\chi_{\mathrm{s}} / t^{\beta}$ versus $x=H / t^{(\gamma+\beta) / 2}$.

Identification of equation (6) with equations (3) and (4) gives :

$$
x_{1}=\gamma ; \quad x_{2}=2 \gamma+\beta ; \quad x_{3}=3 \gamma+2 \beta \text {. }
$$

From the scaling fit values of $\gamma$ and $\beta$, we obtain $x_{1}=3.1 \pm 0.1, x_{2}=7.6 \pm 0.3$ and $x_{3}=12.1 \pm 0.5$ in full agreement with the series expansion fit values. From the scaling function (5) and its series expansion (6) it is clear that $H_{\max }^{2} / t^{\nu+\beta}$ would be constant. Indeed observed in our experiments, this ratio is equal to about $4 \times 10^{7} \mathrm{Oe}^{2}$.

From the scaling expression of $\chi_{\mathrm{s}}$ (Eq. (5)) one obtains the following scaling function for the non linear magnetization :

$$
M_{\mathrm{s}}=\chi_{0}(T) \cdot H-M=t^{\frac{\gamma+3 \beta}{2}} \cdot G\left[\frac{H}{t^{\frac{\gamma+\beta}{2}}}\right]
$$

where $G(x)=\int_{0}^{x} g\left(x^{\prime}\right) \mathrm{d} x^{\prime}$.

At a given field value, when $t \rightarrow 0$, we have $H^{2} / t^{\gamma+\beta} \rightarrow \infty$ but experimentally $M_{\mathrm{s}}$ does not diverge. This implies the following asymptotic form of $G(x)$ for $x \rightarrow \infty: G(x) \sim x^{1+2 \beta /(\gamma+\beta)}$. The asymptotic form of $g(x)$ is thus given by

$$
g(x) \sim x^{2 \beta /(\gamma+\beta)} .
$$

In figure 5 we have represented the experimental data $g(x)=\chi_{\mathrm{s}} / t^{\beta}$ versus $x=\left(H^{2} / t^{\gamma+\beta}\right)^{1 / 2}$ on a $\log -\log$ scale. For small values of $x, g(x)$ is proportional to $x^{2}$. (Eq. (6)) and for high values of $x, g(x)$ seems to tend to the predicted asymptotic form (8), It should be also pointed out that $\chi_{\mathrm{s}}$ does not diverge at the freezing temperature $T_{\mathrm{f}}$. 


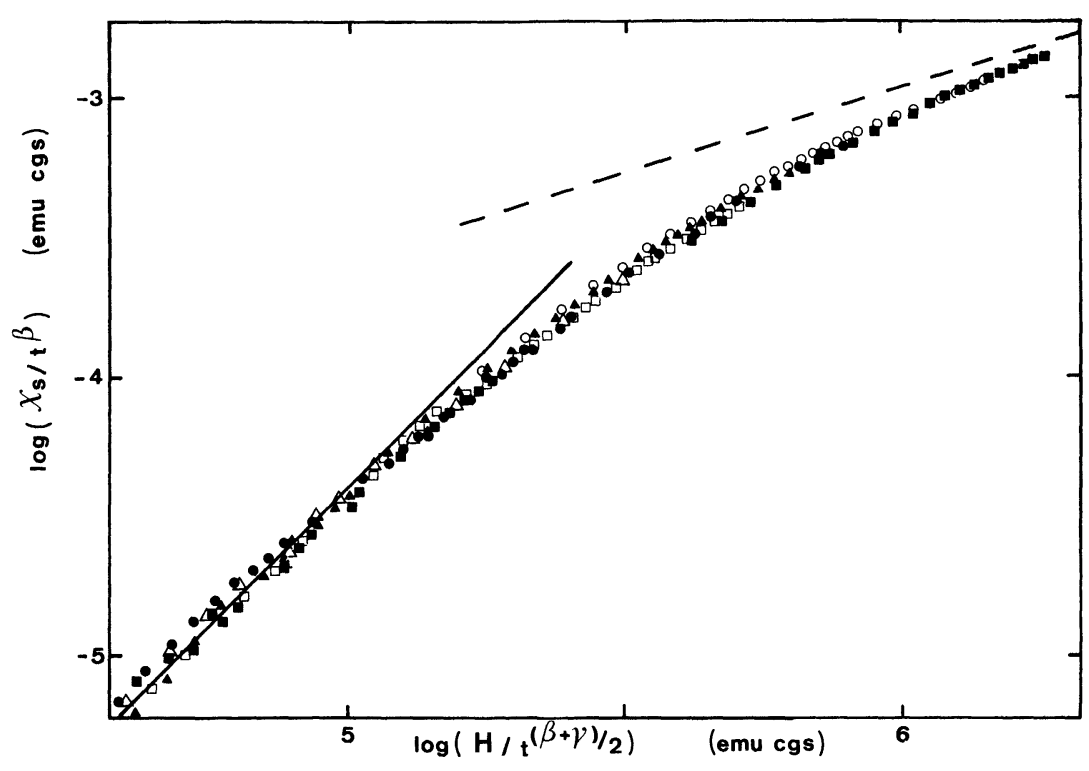

Fig. 5. - Scaling function $g(x)=\chi_{\mathrm{s}} / t^{\beta}$ versus $x=H / t^{(\gamma+\beta) / 2}$ on $\log -\log$ scales. The full line displays the initial slope of $g(x)$; the dotted line shows the asymptotic one $2 \beta / \gamma+\beta$ for $x \rightarrow \infty$.

\section{Static magnetization.}

In order to complete the ac susceptibility data in the close vicinity of the freezing temperature, we have performed magnetization measurements in fields ranging from $10^{-2}$ to 10 Oe with a high sensitivity SQUID magnetometer [5].

3.1 RelaXATION PHENOMENA OF THE Field COOLED MAGNETIZATION (FCM). - Recently, Lundgren et al. have observed time effects of the FCM in AuFe [6]. We have also observed similar relaxation phenomena in $\mathrm{Mn}$ alumininosilicate for fields of $10^{-2}, 10^{-1}$ and $3 \mathrm{Oe}$. Following a step decrease of a few milliKelvin in the temperature of the sample near $T_{\mathrm{f}}$, the FCM first increases very steeply and then exhibits a slow relaxation during several minutes. This relaxation has an opposite direction above and below $T_{0}=2.95 \pm 0.01 \mathrm{~K}$ as shown in figure 6. Above $T_{0}$ the magnetization continues to increase towards its equilibrium values; below $T_{0}$, after the initial increase, the FCM then decreases. The temperature $T_{0}$ at which the relaxation effect vanishes is strikingly equal to $T_{\mathrm{f}}$ determined from the change of slope in the FCM versus $T$ (inset of Fig. 1). This peculiar relaxation phenomena behaviour could provide a very precise determination of $T_{\mathrm{f}}$. No field dependence of this freezing temperature was observed in the experimental field range 0.01-3 Oe.

3. 2 Non LiNEAR MagNetization. - The equilibrium magnetization has been measured in the temperature range $2.9-4.2 \mathrm{~K}$ and particularly near $T_{\mathrm{f}}$, for $t<5 \times 10^{-2}$. The ratios $M / H$ for $H=0.01,0.1$ and $3 \mathrm{Oe}$, were compared. The values of $H$ were adjusted in order to insure the same value of $\frac{M}{H}(T)$ above $3.3 \mathrm{~K}$, where the non linear magnetization is negligible in low fields. Down to $T_{\mathrm{f}}$, there is no difference between the $M / H$ values for $H=0.01$ and 0.1 Oe while a difference for $H=3 \mathrm{Oe}$ is clearly evidenced below $3.1 \mathrm{~K}$ (Fig. 7). This non linear contribution 


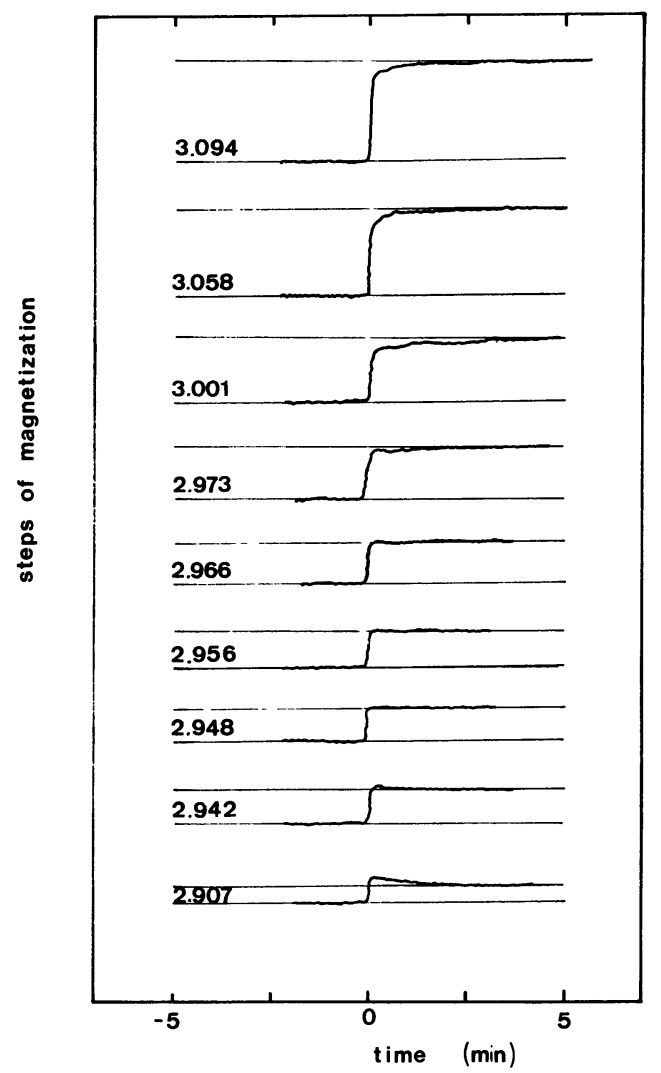

Fig. 6. - Steps of magnetization versus time during a temperature step decrease of $7 \mathrm{mK}$.

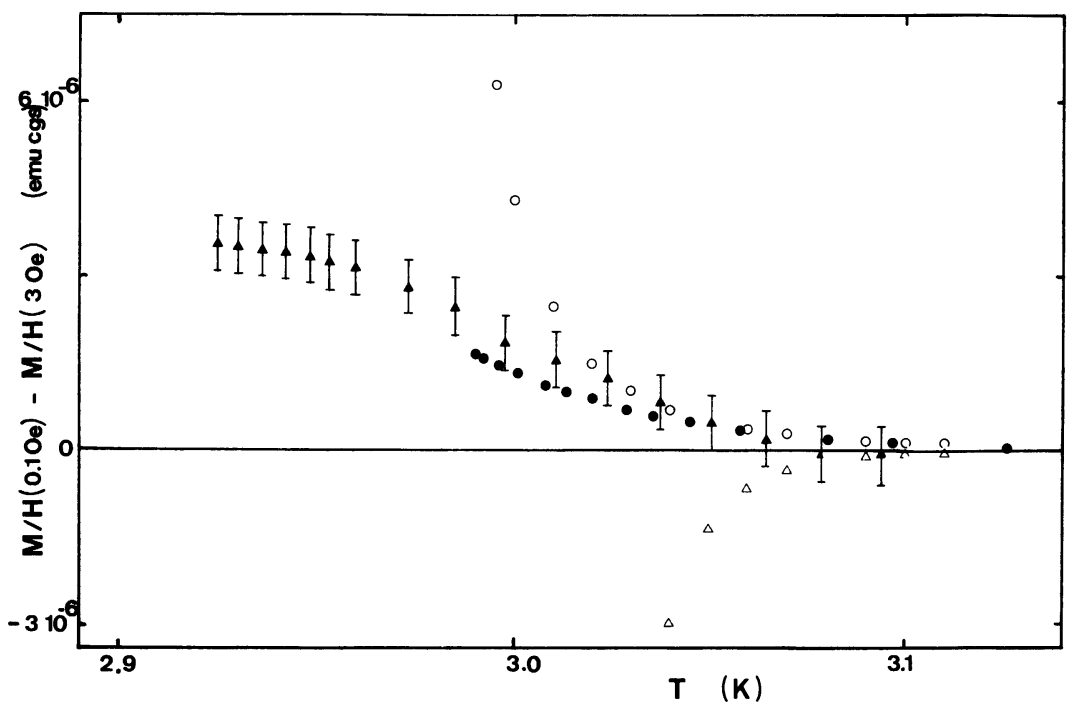

Fig. 7. - Non linear magnetization versus temperature in the close vicinity of $T_{\mathrm{f}} \cdot\left\{\frac{M}{H}(0.1 \mathrm{Oe})-\frac{M}{H}(3 \mathrm{Oe})\right\}$ experimental data are in black triangles. The computed ones (Eq. (7)) are in black circles. Open circles and triangles displays the contribution of the two first terms of series expansion (1). 
increases as temperature decreases and then remains approximatively constant for temperatures lower than $T_{\mathrm{f}}$. We have also shown in figure 7 the two first non linear terms of the field expansion (1) which diverges at $T_{\mathrm{f}}$, and thus cannot describe our data.

Using the experimental scaling function $g(x)$, the ratio $M / H$ for $H=0.1$ and 3 Oe has been computed (Eq. (7)) down to $2.99 \mathrm{~K}$. For $H=0.1$ Oe the computed non linear magnetization is negligible. The computed value for $3 \mathrm{Oe}$ is shown in figure 7. A good agreement with the experimental values is observed. To our knowledge, this is the first experimental check of the validity of the scaling function in the close vicinity of the freezing temperature of a spin glass.

\section{Conclusion.}

The amorphous manganese aluminosilicate, an insulating compound with short-range exchange interactions exhibits a spin glass behaviour with a freezing temperature $T_{\mathrm{f}}=2.95 \mathrm{~K}$. From precise ac susceptibility measurements, we have obtained the non linear part $\chi_{\mathrm{s}}=\chi_{0}-\partial M / \partial H$ of the susceptibility in the reduced temperature range 0.1-0.4. It can be described by the scaling function $\chi_{\mathrm{s}}=t^{\beta} f\left(H^{2} / t^{\gamma+\beta}\right)$ with $\gamma=3.1 \pm 0.1$ and $\beta=1.4 \pm 0.1$. The value of the exponent $\gamma$ characterizing the temperature divergence of the first term of the non linear magnetization is in good agreement with those previously obtained in the metallic spin glasses. Assuming that this scaling function remains valid near $T_{\mathrm{f}}$, the non linear magnetization $M_{\mathrm{s}}=\chi_{0} H-M$ was obtained by numerical integration. It is in fairly good agreement with the SQUID magnetometer measurements in field of 0.1 and 3 Oe. This experiment is the first experimental test of the validity of the scaling function near the freezing temperature of a spin glass.

\section{Acknowledgments.}

The authors wish to thank Dr. Seiden for fruitful theoretical discussion on the order parameter and series expansion of the non linear magnetization in spin glasses and Dr. K. Knorr for providing high quality samples.

\section{References}

[1] Toulouse, G., Gabay, M., J. Physique Lett. 42 (1981) L-103.

[2] Monod, P., Bouchiat, H., J. Physique Lett. 43 (1982) L-45.

[3] Barbara, B., Malozemoff, A. P., Imry, P., Phys. Rev. Lett. 47 (1981) 1852 ; MMM Conf. (Montreal, 1982) J. Appl. Phys. 53 (1982) 7672.

[4] Omari, R., Préjean, J. J., Souletie, J., J. Physique 44 (1983) 1069.

[5] Beauvillain, P., Dupas, C., Renard, J. P. and Veillet, P., Phys. Rev. B (1984) in press.

[6] Lundgren, L., Svedlindh, P., Beckman, O., Phys. Rev. B 26 (1982) 3990. 